\title{
Judgments of lateral distance using transients presented with interaural differences of time
}

\author{
KOUROSH SABERI, DAVID R. PERROTT, and TOKTAM SADRALODABAI \\ California State University, Los Angeles, California
}

\begin{abstract}
Measurements of perceived lateral distance were made using transients in a lateralization paradigm. Two binaural clicks (referred to as boundary clicks) presented with interaural differences of time (IDT) were used to indicate a lateral "distance" along the interaural axis. IDT for the first click was zero (diotic) and for the second, a fixed and predetermined value (dichotic). The location of an additional click (pointer click) was controlled by the subjects, who were instructed to bisect the perceived lateral distance between the two boundary clicks with this pointer click. The procedure was repeated for three distances. Results indicate a linear relation between judgments of distance and changes in IDT $\left(r^{2}=0.999\right)$.
\end{abstract}

Research on auditory spatial function has primarily involved lateralization paradigms, since they allow greater stimulus control and a much more manageable experimental environment. Using such paradigms, researchers have studied the effects of various interaural parameters on the intracranial displacement of auditory images along the binaural axis (see Yost \& Hafter, 1987). In reviewing this literature, however, we have encountered certain important assumptions with regard to the relation between different sensory spaces and the capacities of the observer to adopt various frames of references. The most significant of these assumptions is the idea that auditory space can be unambiguously mapped onto nonauditory space, which has led to the wide use of nonauditory spatial systems in describing auditory localization. An example is the work of Jeffers and Taylor (1961), who report using a suspended semicircle of lights positioned six feet from the subject to make measurements of the location of intracranial images. Other paradigms have included tactile referents (e.g., a puff of air on the skin of the forehead; Békésy, 1960) and various other visual maps (Hafter, Bourbon, Blocker, \& Tucker, 1969; Sayers, 1964; Teas, 1962; Yost, 1981; Yost, Tanis, Nielsen, \& Bergert, 1975). Such intermodal paradigms are rarely, if ever, encountered in nonspatial psychoacoustic research or in other nonauditory spatial research, such as on visual space (see Lunburg, 1950; Wagner, 1985). It would be inconceivable, for example, to envision the mapping of visual space by referencing a known distribution of sound images. Other assumptions include those of response immunity to mental rotations from one perspective (e.g., intra-

This research was supported in part by grants awarded to the second author from the National Science Foundation (BNS-8512317) and the National Institutes of Health (3S06-RR0801-1452). Correspondence should be addressed to Kourosh Saberi, Department of Psychology, University of California, Berkeley, CA 94720.
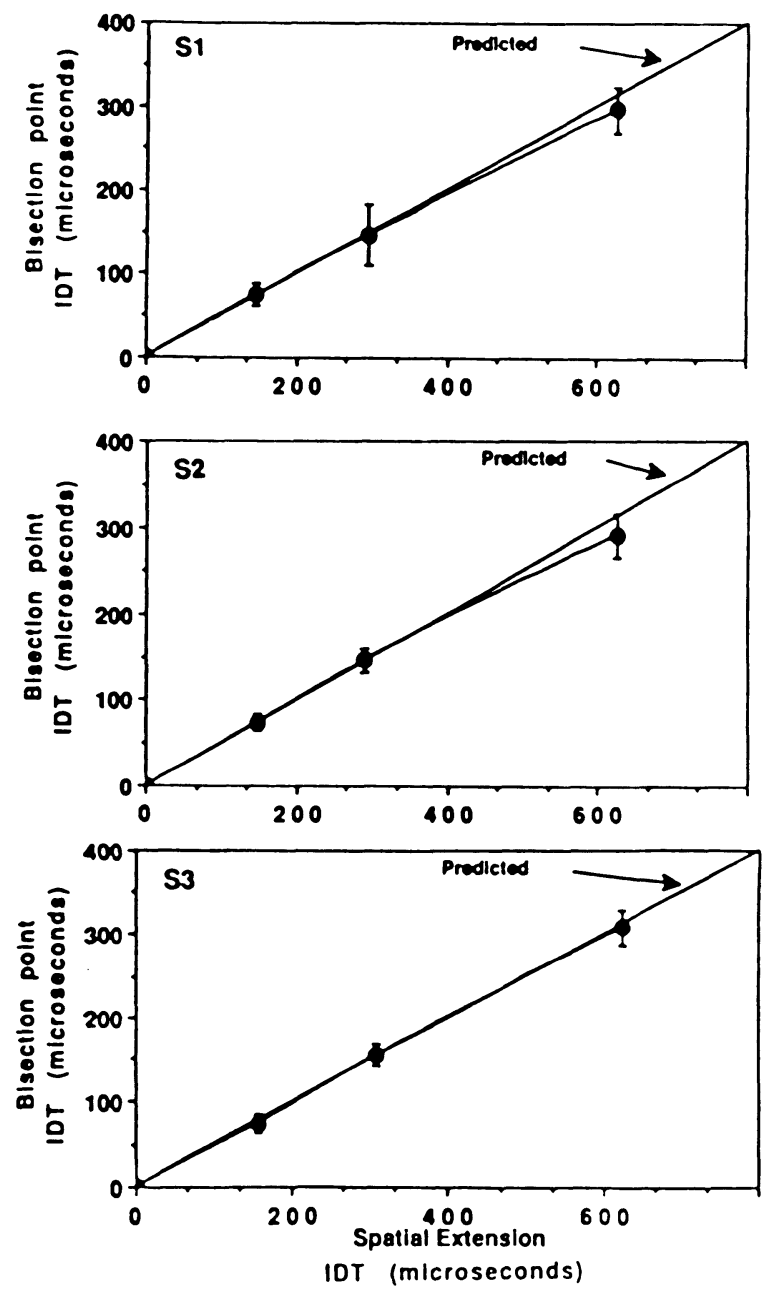

Figure 1. Plot of the bisection points as a function of the distance to be bisected (in terms of IDT separation) for three subjects. The straight line is a linear fit ( 0.5 slope). Each symbol (filled circle) represents 40 to 50 estimates of the bisection point. Each error bar represents one standard deviation. 
cranial) to another (e.g., top-down), the external projection of images, and the translation into scales of differing magnitudes (Jeffers \& Taylor, 1961). More recently, in an attempt to avoid such transformations, Bernstein and Trahiotis (1985), Trahiotis and Bernstein (1986), and Trahiotis and Stern (1989) have employed acoustic pointers based on interaural differences of intensity (IDI) to match the position of lateralized images. However, no attempt has been made to directly obtain a metric of lateral space using such intracranial pointers. In our search for a procedure by which we could both study the spatial organization of lateralization and avoid unnecessary transformations, we have come across the simple bisection paradigm, which requires neither crossmodal translations nor changes in the frame of reference of the observer. In the present experiment, an acoustic pointer is used to bisect a lateral distance whose boundaries are marked off by two other auditory events. Previous studies of this type (Yost, 1981; Békésy, 1960) have reported a linear relation between interaural parameters and the perceived location of auditory events. ${ }^{1}$ Should the auditory spatial function be immune to the methods mentioned above, one would expect to obtain a similar linear relation in the present study.

\section{METHOD}

\section{Subjects}

Three university students served as subjects. Two (S1, S2) had extensive experience in psychoacoustic experiments, including lateralization paradigms, and were given a single practice session before data collection began. The third subject (S3) underwent several weeks of practice.

\section{Apparatus}

Signals were generated by a microprocessor whose output was directed to a pair of multifunction signal generators (Wavetek Model 184). The output of each generator was then fed directly into earphones (TDH 49). Pulses thus generated were $40-\mu \mathrm{sec}$ square waves. ${ }^{2}$ Signal level was set at $55 \mathrm{~dB}$ (A-weighted). The intracranial acoustic pointer was a dichotic binaural click whose interaural difference in time (IDT) was controlled by the subject with a sensor pad (Koala Model 202). IDTs varied as a linear function of the location indicated. For example, pressure on the extreme right portion of the sensor pad produced an IDT of $640 \mu \mathrm{sec}$ (right ear leading). With the use of this technique, IDTs could be instantaneously varied in steps as large as $640 \mu \mathrm{sec}$ and as small as $2.5 \mu \mathrm{sec}$, starting from a minimum of $15 \mu \mathrm{sec}$.

\section{Procedure}

Each trial involved the presentation of a three-click sequence. The first click was always diotic (IDT $=0$ ), the second, dichotic (with the right ear leading), and the third also dichotic, leading to the same side as the second click (e.g., $625 \mu \mathrm{sec}$ in the initial session). The subjects were instructed to bisect the distance between the first and third clicks with the second (pointer) click. This three-click sequence was repeated
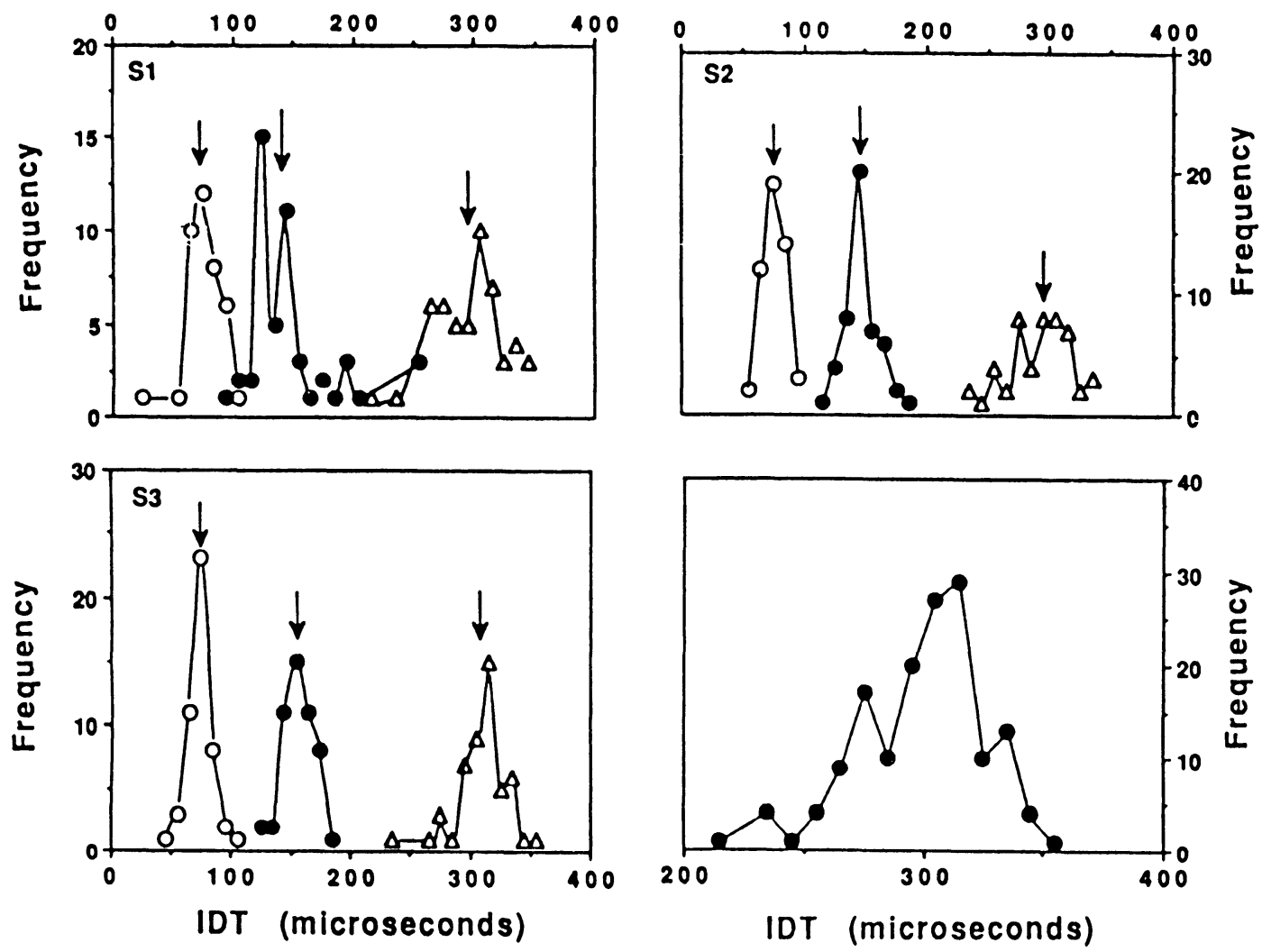

Figure 2. Modal responses (symbols) for the three distances plotted against mean responses (vertical arrows) for three subjects. Each modal point represents responses within a $10-\mu \mathrm{sec}$ increment. The lower right panel is the pooled response for the three subjects for the largest distance to be bisected (see text). 
continuously with a one-sec intersequence interval until the subject judged the pointer to be at the bisection point. They then pressed a key to record its IDT (each subject, on the average, made approximately 10 adjustments in the location of the pointer before making a final judgment). The location of the acoustic pointer was randomized at the beginning of each new recording along the entire range of all possible IDTs. Ten recordings constituted one session of the experiment. Four to five sessions ( 40 to 50 recordings of the bisection point) were completed by each subject for each distance. Subjects completed a maximum of only two sessions per day. The mean IDT corresponding to this bisection point was then taken to define the new distance (i.e., from the median plane to this point), and the bisection task was continued for another five sessions until the second bisection point was obtained. This procedure was repeated to obtain a third bisection point for a third distance.

\section{RESULTS AND DISCUSSION}

The data for three subjects are plotted in the three panels of Figure 1. These results suggest a generally linear relation between the perceived lateral distance and IDTs for transients (mean $\left.r^{2}=0.999\right)$. A slight negative acceleration is evident for 2 of the 3 subjects. Statistical analysis, however, showed no significance of this latter slope. Data variability generally increases with increasing distance from the median plane (with the exception of the second bisection point for $\mathrm{S} 1$ ). Further analysis suggests that this increase in error is most likely due to increasing the relative distances (i.e., constant percent error). The straight line in Figure 1 is a linear fit (0.5 slope), and error bars represent one standard deviation. Post hoc experiments showed no significant order effects. Similar results were observed when the order of presentation of the three-click sequence was reversed.

The four panels of Figure 2 are a plot of the response frequencies grouped in $10-\mu \mathrm{sec}$ increments (modal responses). The arrows represent the mean for each group of responses (i.e., each distance to be bisected). The lower right panel is the pooled response frequency for the largest continuum $(0-625 \mu \mathrm{sec})$. Data from other continuums could not be pooled, since subsequent distances were different for each subject. Yost (1981) has suggested different distributions for plots of modal and mean responses for phase disparities. The present data, however, demonstrate good agreement between the mode and mean distributions for the smallest distance (open circles). Fluctuation increases for the larger distances. Mean and modal points depart as responses are made farther from the median plane.

Finally, our results seem to support the notion outlined earlier that localization performance is relatively immune to various transformations, at least to the extent employed in previous experiments. ${ }^{3}$ This is evident from the nearly perfect linear function obtained in the present study. That no significant differences were observed between our results and previous work (Teas, 1962; Yost, 1981; Yost et al., 1975) suggests that human observers are capable of translating spatial information across various sensory channels (visual or tactile) or of adopting arbitrary changes in their frame of reference without substantially altering the content of this information.

\section{REFERENCES}

BékÉsy, G. von (1960). In E. G. Wever (Ed. and Trans.), Experiments in hearing. New York: McGraw-Hill.

BernsteIn, L. R., Trahiotis, C. (1985). Lateralization of lowfrequency, complex waveforms: The use of envelope-based temporal disparities. Journal of the Acoustical Society of America, 77, 1868-1880.

Hafter, E. R., Bourbon, W. T., Blocker, A. S., \& Tucker, A. (1969). A direct comparison between lateralization and detection under conditions of antiphasic masking. Journal of the Acoustical Society of America, 46, 1452-1457.

JefFers, L. A., \& TAYLOR, R. W. (1961). Lateralization vs. localization. Journal of the Acoustical Society of America, 33, 482-483.

LUNBURG, R. K. (1950). The metric of binocular visual space. Journal of the Optical Society of America, 40, 627-642.

SAYERS, B. (1964). Acoustic-image lateralization judgments with binaural tones. Journal of the Acoustical Society of America, 36, 923-926.

TEAS, D. C. (1962). Lateralization of acoustic transients. Journal of the Acoustical Society of America, 34, 1460-1465.

Trahiotis, C., \& Bernstein, L. R. (1986). Lateralization of bands of noise and sinusoidally amplitude-modulated tones: Effects of spectral locus and bandwidth. Journal of the Acoustical Society of America, 79, 1950-1957.

Trahiotis, C., \& Stern, R. M. (1989). Lateralization of bands of noise: Effects of bandwidth and differences of interaural time and phase. Journal of the Acoustical Society of America, 86, 1285-1293.

W Agner, M. (1985). The metric of visual space. Perception \& Psychophysics, 38, 483-495.

Yost, W. A. (1981). Lateral position of sinusoids presented with interaural intensive and temporal differences. Journal of the Acoustical Society of America, 70, 397-409.

Yost, W. A., HAfTer, E. R. (1987). Lateralization. In W. A. Yost \& G. Gourevitch (Eds.), Directional Hearing. New York: SpringerVerlag.

Yost, W. A., TANis, D. C., Nielsen, D. W., Bergert, B. (1975). Interaural time versus interaural intensity in a lateralization paradigm. Perception \& Psychophysics, 18, 433-440.

\section{NOTES}

1. An exception is the modal distribution for interaural phase disparity (see Yost, 1981).

2. Transients were selected for their wide use in binaural research and for their relatively sharp image. Note that the parameter was interaural time disparity and not phase.

3. The paradigm employed here most resembles that of Békésy's in that we have used transients presented with IDTs. Other researchers have obtained similar results despite using various other types of signals (e.g., sinusoids) and interaural parameters (e.g., phase and intensity).

(Manuscript received July 20, 1990.) 